\title{
Refractive Error and or Visual Impairment and Their Impact on Quality of Life Among School-age Children in Sub-saharan Africa; A Protocol of a Systematic Review
}

Tshubelela Sello Simon Magakwe ( sellomagakwe80@gmail.com )

University of KwaZulu-Natal - Westville Campus https://orcid.org/0000-0002-8724-9911

Zamadonda Nokuthula Queen Xulu-Kasaba

University of KwaZulu-Natal College of Health Sciences

Rekha Hansraj

University of KwaZulu-Natal College of Health Sciences

\section{Research Article}

Keywords: Refractive error, Visual impairment, Quality of Life, child eye health, sub-Saharan Africa, systematic review

Posted Date: May 10th, 2021

DOl: https://doi.org/10.21203/rs.3.rs-500252/v1

License: (c) (1) This work is licensed under a Creative Commons Attribution 4.0 International License.

Read Full License 


\section{Abstract}

\section{Background}

Refractive error (RE) is a leading cause of visual impairment $(\mathrm{VI})$ in children, the most common cause of childhood handicaps, and the second leading cause of childhood blindness globally. Refractive error and/or VI limits the ability of a child to perform well at school, in most sporting activities which require good vision, and negatively affects the socio-economical security of an individual. These two conditions have also reported to impact quality of life (QoL). The epidemiological evidence about the prevalence and distribution of RE and $\mathrm{VI}$, as well their impact on the QoL of school-going children in Sub-Saharan Africa (SSA), will assist policymakers and stakeholders involved in child eye care in channelling resources appropriately. The main objective of this scoping review study therefore is to map available evidence on RE and or VI and their impact on QoL of school-going children in SSA.

\section{Methods}

This systematic review study will follow the five steps outlined in a framework proposed by Arksey and O'Malley (2005). The literature search will be conducted using the following databases, EBSCOhost: CINAHL, Academic search complete, Health-Consumer and Health-Nursing, Google Scholar, Science direct and PubMed. The period of the literature will be from January 1998 to 31 December 2020, all suitable studies will be exported to a Mendeley library and in cases of outstanding articles the authors will be contacted directly. The title screening will be done by the principal investigator ( $\mathrm{PI})$, then the study will employ two independent reviewers which will have access to the Mendeley library to do abstract screening (AS) and a full article screening (FAS). Following FAS, data extraction will be done by PI. The quality index of all included studies will be determined using the Mixed Method Appraisal Tool (MMAT). The results will be reported using the Preferred Reporting Items for systematic reviews and Meta-Analyses (PRISMA).

\section{Discussion}

The evidence of this scoping review will guide policy makers and all stakeholders in planning intervention strategies to address this global issue. Furthermore, the results of this review will be published in a peer reviewed journal and will be used to guide future research in this and related areas.

\section{Systemic review registration: Submitted (PROSPERO) 27/04/2021}

\section{Background}

Normal vision is essential in the general development of a child and serves as a major sensory modality in humans.(1) Any disturbance to vision therefore can affect the development and the learning of an individual negatively, worse so if the visual deficit exists at birth or in an early stage of life.(2) Refractive error is currently a leading cause of visual impairment $(\mathrm{VI})$ in children, the most common cause of 
childhood handicaps, and the second leading cause of childhood blindness. $(3,4)$ Refractive error and/or VI limits the ability of a child to perform well at school as it is known that $85 \%$ of learning depends on vision. Moreover, affected children cannot participate in most sporting activities which require good vision. The ultimate negative effect however is on the socio-economic security of affected individuals.(5) (6) Thus, not surprisingly, refractive error (RE) and or VI has been reported to impact the quality of life (QoL). (5) Furthermore, managing the impact of $\mathrm{VI}$ and blindness from uncorrected RE (URE) is costlier and puts more pressure on the global economy than managing RE itself. (7) Globally there are around 19 million visually impaired children (8) of which 12.8 million are due to uncorrected or inappropriately corrected RE with eight million children being classified as blind due to URE.(9) Ninety percent of children with VI were found to reside in the developing countries. $(10,11)$

The impact of RE and $\mathrm{VI}$ onset before or during school-going age is significantly greater as compared to onset in adulthood, considering the greater number of years that they will likely carry these conditions in the case of the former.(1) The World Health Organization (WHO) developed a policy more than two decades ago in 1998 called the "WHO global school initiative: Helping schools to became 'HealthPromoting Schools' " and management of VI was included.(12) Two years later at the global meeting convened by the $\mathrm{WHO}$ in London, the recommendation that further information was required on the prevalence, incidence, and causes of VI among school-going children especially in the developing countries, emanated.(13) Further, in the initiation of 'VISION 20/20 RIGHT TO GOOD SIGHT' by the WHO and other non-government organizations (NGOs), the management of RE and VI in children was prioritized.(14) Knowledge of the magnitude and prevalence of RE and/or VI among school-going children therefore is vital for the proper channeling of resources for the management of these conditions. Moreover, it has been reported that RE and VI differ according to ethnic groups. $(15,16)$ There is therefore a need for further research to be conducted to improve on the current knowledge and understanding of the epidemiology of these conditions which could facilitate future strategies to minimize the risks and manage it.

Epidemiological evidence about the prevalence and distribution of RE and $\mathrm{VI}$, as well as their impact on the QoL of school-going children in Sub-Saharan Africa, will assist policymakers and stakeholders involved in child eye care in channeling resources appropriately. The objective of this scoping review study therefore is to map available evidence on RE and or VI and their impact on the QoL of school-age children in Sub-Sahara African (SSA).

\section{Research question}

What is the prevalence and distribution of RE and $\mathrm{VI}$ in school-age children in SSA, and the impact of these on their QoL?

\section{Methodology}

This review will map available evidence for SSA on RE and VI, as well as their impact on the QoL of school-age children. This systematic scoping review will follow five steps outlined in a framework 
proposed by Arksey and O'Malley.(17) The steps are as follows (1) Identify the research question, (2) Identify relevant studies, (3) Study selection, (4) Charting the data, and (5) Collating, summarizing and reporting the results.

\section{- Identifying the research question}

The research question has been identified as: "What is the prevalence and distribution of RE and VI in school-age children in SSA?

Sub-Question: What impact do these have on QoL?

\section{Eligibility criteria}

Studies to be included:

- Studies reporting on RE

- Studies reporting on VI

- Studies showing evidence on the impact of RE on QoL

- Studies showing evidence on the impact of VI on QoL

- Those with participants from ages 5 to 20 years of age

- Subjects being children of school age

- Studies conducted on the SSA countries

- Peer-reviewed black literature

- Peer-reviewed grey literature

- Studies published in the period 1998 to 2020

\section{Exclusion criteria:}

All studies not published in English, all qualitative studies and all studies that are not primary studies, will be excluded.

The Population Concept Context (PCC) framework will be employed in this study (Table 1).

TABLE 1: Population Concept Context framework

\begin{tabular}{|ll|}
\hline PCC & Detailed \\
\hline Population & school-going children/ learners/pupil \\
\hline Concept & Refractive error OR visual impairment AND quality of life \\
\hline Context & Sub-Sahara Africa \\
\hline
\end{tabular}




\section{Identifying relevant studies}

All peer-reviewed literature and any grey literature accessible online will be included in this systematic review. This review will focus on four main areas of interest, which are RE, VI, QoL and school-going children, however not all authors refer to them in these aforementioned terms, therefore we will then use Medical subject heading (MeSH) terms as follows "Refractive error OR Visual impairment OR Ametropia AND Quality of Life AND School going children OR Learner OR Scholar OR Pupil”. To identify relevant studies, an electronic search will be conducted in various databases using the keyword search

((("Refractive error OR Visual impairment OR Ametropia AND Quality of life AND school going children OR learner OR scholar OR pupils AND sub-Saharan Africa")))

This study search will be conducted using the following databases:

- Google Scholar

- Science direct

- PubMed

- EBSCOhost: CINAHL, Academic search complete, Health-Consumer and Health-Nursing.

The articles found will be exported to a Mendeley library especially created for this study. Thereafter, duplicates will be removed. Table 2 indicates the results of a pilot search. Figure 1 show the screenshot of pilot study done using Science Direct search engine as an example.

TABLE 2: Results of pilot search

\begin{tabular}{|c|c|c|c|}
\hline Keywords searched & $\begin{array}{l}\text { Date of } \\
\text { search }\end{array}$ & $\begin{array}{l}\text { Search } \\
\text { engine } \\
\text { used }\end{array}$ & $\begin{array}{l}\text { Number of } \\
\text { retrieved } \\
\text { publications }\end{array}$ \\
\hline \multirow{4}{*}{$\begin{array}{l}\text { ((("Refractive error OR Visual impairment OR Ametropia } \\
\text { AND Quality of life AND school going children OR learner } \\
\text { OR scholar OR pupils AND Sub-Saharan Africa"))) }\end{array}$} & \multirow{4}{*}{$\begin{array}{l}24 \\
\text { November } \\
2020\end{array}$} & $\begin{array}{l}\text { Google } \\
\text { scholar }\end{array}$ & 667 \\
\hline & & $\begin{array}{l}\text { Science } \\
\text { direct }\end{array}$ & 31561 \\
\hline & & PubMed & 21 \\
\hline & & EBSCOhost & 441570 \\
\hline
\end{tabular}

\section{Study selection}

Suitable studies that are aligned with the inclusion criteria will be exported to a Mendeley library specifically created for this study done by The Principal investigator (PI), after which all duplicates will be removed. This will be followed by abstract screening (AS), conducted independently by two reviewers, 
using a tool developed and piloted for this study (Table 3). Full article screening (FAS) will follow, also conducted independently by two independent reviewers using a tool as (Table 4).

TABLE 3: Abstract Screening tool

1. Author and date:

2. Title:

3. Does this article bear evidence on refractive error/ visual impairment? Yes/No/ Can't tell

4. Does this article bear evidence on school age children/learners (aged 5-20)? Yes/No/ Can't tell

5. Was the study done in Sub-Saharan Africa? Yes/NO/Can't tell

6. Screener - Initials:

TABLE 4: Full Article screening tool

1. Author and date:

2. Title:

3. Does this article bear evidence on refractive error/ visual impairment? Yes/No

4. Does this article bear evidence on school age children/learners (aged 5-20)? Yes/No 1. Was the study done in Sub-Saharan Africa? Yes/No

6. Screener - Initials:

All discrepancies will be resolved by the two screeners meeting to reconcile and agree on those studies they initially differed upon. The University of KwaZulu-Natal (UKZN) library will be the primary source for retrieving articles should the authors not respond to such requests. The process of article selection will be reported using the Preferred Reporting Items for Systematic reviews and Meta-Analyses (PRISMA), as per Figure 2. Following finalizing of articles, the PI will conduct data extraction on all included articles.

\section{Quality assurance of the study}

The quality index of all included studies will be determined using the Mixed Method Appraisal Tool (MMAT). This study will employ the 2018 MMAT tool in conjunction with the 2011 version of MMAT tool. The 2018 tool does not provide actual score yet advises that qualitative analysis be provided rather. However, since this does not have absolute values, the scoring section from the 2011 tool will be adopted with five criteria from 2018 tool to grade the studies. The scoring ranges from $100 \%$ being a good area of study and $20 \%$ being low quality. Two independent reviewers will grade the quality of each study's aim, design, methodology and statistical analysis 


\section{- Charting the data}

An electronic data collection tool to chart evidence that seeks to answer the research question, as shown in Table 5 will be utilized. This tool will extract the necessary information based on prevalence of RE and or VI, distribution of RE and or VI and any impact of RE and or VI on the quality of life. This electronic screening tool will be updated as the need arises during the course of the study.

TABLE 5:Data Electronic collection tool 
1. Author \& Date

2. Article title

3. Country of study

4. Region of study

5. Total sample size

6. Age

7. No of male participants

8. No of female participants

9. No. of participants with $\mathrm{V} / \mathrm{A}$ of $6 / 9$ and worse in the $\mathrm{Re}$

10. No. of participants with V/A of $6 / 9$ and worse in the Le

11. No. of participants with V/A of $6 / 12$ to $6 / 18$ in one or both eye

12. No. of participants with $\mathrm{V} / \mathrm{A}$ worse than $6 / 18$ to $6 / 60$ in one or both eyes

13. No. of participants with $\mathrm{V} / \mathrm{A}$ worse than $6 / 60$ to 3.60 in one or both eyes

14. No. of participants with V/A worse than $3 / 60$ or Visual field loss of 10 degrees or less (Blindness)

15. No. of participants with Visual field loss of 20 degrees to $>10$ degrees

16. Causes of VI

17. No. of participants with RE

18. No. of participants with Myopia

19. No. of participants with Hyperopia

20. No. of participants with Astigmatism

21. No. of male participants with of RE

22. No. of female participants with RE

23. No. of male participants with myopia

24. No. of female participants with myopia

25. No. of male participants with hyperopia

26. No. of female participants with hyperopia

27. No. of male participants with astigmatism

28. No. of female participants with astigmatism

29. Any evidence on the quality of life (QoL)? 
No. = number, $V / A=$ visual acuity, $R E=$ refractive error, $R e=$ right eye,$V I=$ visual impairment,$Q o L=$ quality of life

\section{- Collating, summarizing and reporting the results}

The findings of this systematic scoping review will be captured onto Microsoft Excel and analysed with the help of a statistician. They will thereafter be published in peer-review journals.

\section{Discussion}

The sudden increase in the prevalence of RE in school-going children has been noted recently, especially with the introduction of Edu-comp smart classes in school increasing the use of laptops, as well as increased indoor activities like watching television, using computers and playing mobile phone games. (19) This is the age group that carries the burden of RE and VI for a longtime as compared to older age groups and they are affected socially and economically.(20) As highlighted earlier, out of 19 million visually impaired children globally, $90 \%$ are residing within the developing countries.(21) Sub-Saharan Africa was selected for this review because it consists of the majority of developing countries globally. (21) Therefore, the evidence of this scoping review will guide policy makers and all stakeholders in planning intervention strategies to address this public health concern in low to middle income countries within SSA. To the best of my knowledge, no systematic review or scoping review studies on RE and $\mathrm{VI}$, as well as their impact on the QoL of school-going children in SSA, has been conducted previously.

\section{Abbreviations}

RE: Refractive Error

V/A: Visual Acuity

Vl: Visual Impairment

V/F: Visual Field

QoL: Quality of Life

SSA: Sub-Sahara Africa

WHO: World Health Organisation

LMICs: Low-and Middle-Income Countries

PCC: Population Concept Context 
MeSH: Medical Subject Heading

PRISMA: Preferred Reporting Items for Systematic Review and Meta-Analysis Protocols

MMAT: Mixed Method Appraisal Tool

UKZN: University of KwaZulu-Natal

\section{Declarations}

\section{Ethical and dissemination}

All the data to be used for this scoping review is currently available in the public domain, therefore there is no need for an ethical application or ethical approval.

\section{Ethics approval and consent to participate}

All the data to be used for this scoping review is currently available in the public domain, therefore there was no need for an ethical application or ethical approval neither participants consent.

\section{Consent for publication}

Not applicable

\section{Availability of data and materials}

Data is available from the corresponding author upon the request

\section{Competing interest}

The authors declares that the are no conflict of interests

\section{Funding information}

All authors are equally responsible for all publication costs of this manuscript and no other funding to disclose

\section{Authors' contributions}

TSS, ZNQ and R conceptualize the study, TSS drafted the protocol, ZNQ and R facilitate and edit the final manuscript, TSS act as the corresponding author and submit the manuscript for publication purpose.

\section{Disclaimer}

The expressed view on this manuscript are those of authors and not necessarily reflect the official policy of the University of KwaZulu-Natal. 
Acknowledgements

We would like to acknowledge Ms Samkelo Xulu for helping with the screening

\section{References}

1. Rahi JS, Dezateux C. Epidemiology of visual impairment in Britain. Archives of Disease in Childhood BMJ Publishing Group. 1998;78:381-6.

2. Atowa UC, Hansraj R, Wajuihian SO. Visual problems: a review of prevalence studies on visual impairment in school-age children. Int J Ophthalmol. 2019;12(6):1037-43.

3. Dandona L, Dandona R, Srinivas M, et al. Blindness in the Indian state of Andhra Pradesh. Investig Ophthalmol Vis Sci. 2001;42:908-16.

4. Al Wadaani FA, Amin TT, Ali A, et al. Prevalence and pattern of refractive errors among primary school children in Al Hassa, Saudi Arabia. Glob J Health Sci. 2012;5(1):125-34.

5. Falkenberg HK, Langaas T, Svarverud E. Vision status of children aged 7-15 years referred from school vision screening in Norway during 2003-2013: A retrospective study. BMC Ophthalmol. 2019;19(1):180.

6. Resnikoff S, Pascolini D, Mariotti SP, et al. Global magnitude of visual impairment caused by uncorrected refractive errors in 2004. Bull World Health Organ. 2008;86:63-70.

7. Vitale S, Cotch MF, Sperduto R, et al. Costs of Refractive Correction of Distance Vision Impairment in the United States, 1999-2002. Ophthalmology. 2006;113(12):2163-70.

8. Naipal S, Rampersad N. A review of visual impairment. African Vis Eye Heal. 2018;77(1):4.

9. Prevention of Blindness and Deafness. Available data on blindness, update 2006. World Health Organisation Geneva; 2005.

10. Dandona R, Dandona L, Srinivas M, et al. Refractive error in children in a rural population in India. Investig Ophthalmol Vis Sci. 2002;43:615-22.

11. Adeoti CO. Beliefs and attitude towards spectacles. Niger J Clin Pract. 2009;12(4):359-61.

12. WHO's global school health initiative. Health promoting schools. Geneva: World Health Organization; 1998.

13. Prevention of childhood blindness. World Health Organisation, Geneva 1992.

14. WHO I. What is VISION 2020? World Health Organisation; 2020.

15. Soler M, Anera RG, Castro JJ, et al. Prevalence of refractive errors in children in Equatorial Guinea. Optom Vis Sci. 2015;92(1):53-8.

16. French AN, Morgan IG, Burlutsky G, et al. Prevalence and 5- to 6-Year Incidence and Progression of Myopia and Hyperopia in Australian Schoolchildren. Ophthalmology. 2013;120(7):1482-91.

17. Arksey H, O'Malley L. Scoping studies: Towards a methodological framework. Int J Soc Res Methodol Theory Pract. 2005;8(1):19-32. 
18. Moher D, Liberati A. Preferred reporting items for systematic reviews and meta-analyses: the PRISMA statement (Chinese edition). jhu.pure.elsevier.com 2009.

19. Pradhan N, Sachdeva A, Goel T, et al. Prevalence of refractive errors among school children of 6-12years of age group and reason for not using spectacles even after correction. Int J Res Med Sci. 2018;6(3):798.

20. Rahi JS, Solebo AL, Cumberland PM. Uncorrected refractive error and education. BMJ. 2014;349(3):5991-1.

21. Pascolini D, Mariotti SP. Global estimates of visual impairment: 2010. Br J Ophthalmol. 2012;96(5):614-8.

\section{Figures}

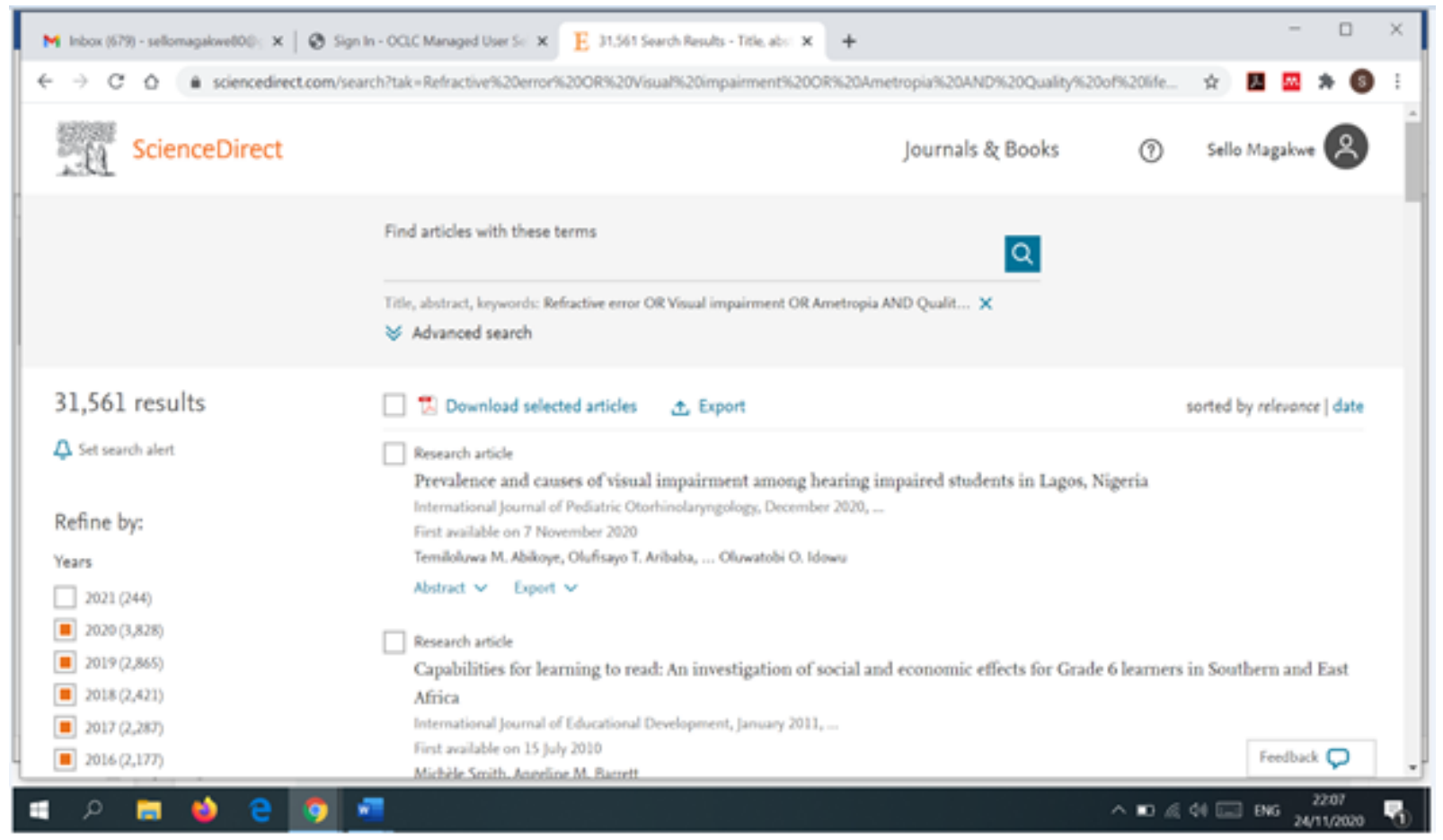

\section{Figure 1}

Screenshot of pilot study in Science Direct by the author 

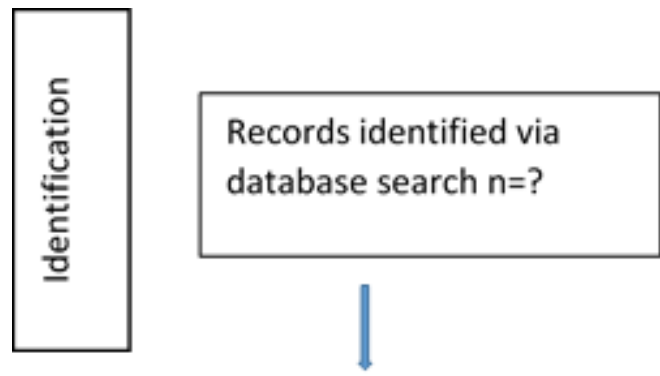

Additional records identified via other sources $n=$ ?
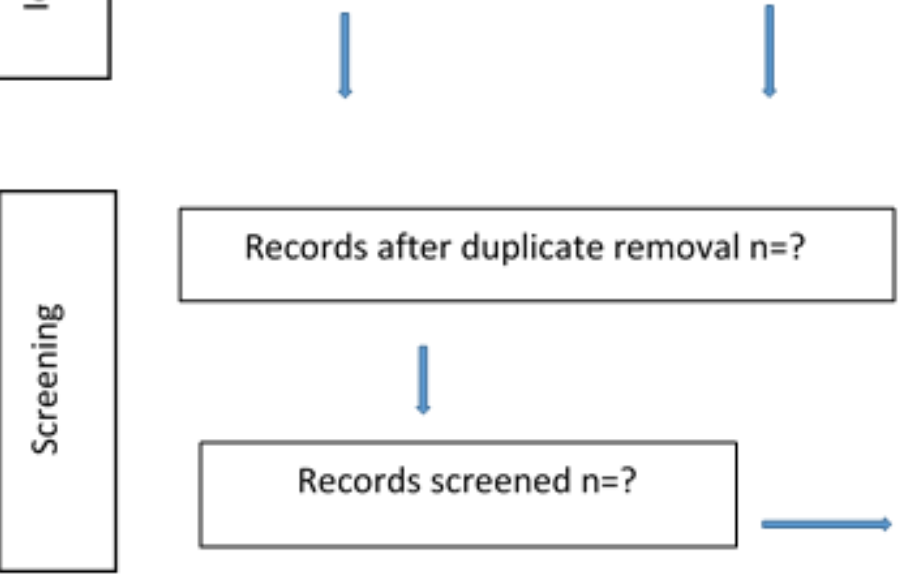

Records excluded $n=$ ?
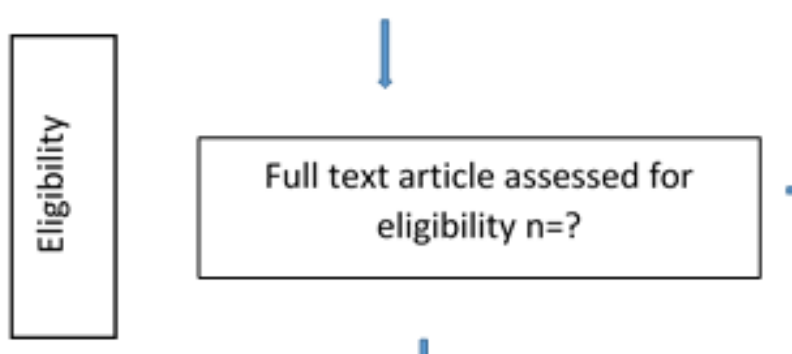
Full text article assessed for eligibility $n=$ ?

Full text article excluded with reason $\mathrm{n}=$ ?

\section{Studies included in synthesis $n=$ ?}

Figure 2

Preferred Reporting Items for Systematic Review and Meta-Analysis Protocols (PRISMA) flow diagram (Source adopted from (Moher et al., 2009). ( $n=$ number)

\section{Supplementary Files}

This is a list of supplementary files associated with this preprint. Click to download.

- PRISMAPchecklistBMC2021.docx 